\title{
Electrochemical Impedance Spectroscopy Studies of HF CVD Diamond Films
}

\author{
M. Kowalska ${ }^{a}$, K. FAbisiak ${ }^{b, c}$, A. Wrzyszczyński ${ }^{a}$, M. Szybowicz ${ }^{d, *}$ And K. PAProcki ${ }^{b}$ \\ ${ }^{a}$ Faculty of Chemical Technology and Engineering, University of Technology and Life Sciences, \\ Seminaryjna 3, 85-326 Bydgoszcz, Poland \\ ${ }^{b}$ Institute of Physics, Kazimierz Wielki University, Powstańców Wielkopolskich 2, 85-090 Bydgoszcz, Poland \\ ${ }^{c}$ Medical Physics Department, Oncology Center, I. Romanowskiej 2, 85-796 Bydgoszcz, Poland \\ ${ }^{d}$ Faculty of Technical Physics, Poznań University of Technology, Piotrowo 3, 60-965 Poznań, Poland
}

(Received February 26, 2015; in final form August 13, 2015)

\begin{abstract}
Diamond films were synthesized by a hot filament vapor deposition method using $\mathrm{H}_{2} / \mathrm{CH}_{4}$ gas mixtures. A Hioki impedance analyzer was used to study the dielectric properties of the deposited diamond films. The dielectric dispersion measurement yielded the real and imaginary parts of impedance of diamond films in the form of a Nyquist plot in a complex plane. The obtained results were fitted by using equivalent circuit which consists of three impedance $(Z)$ components containing resistor $R$ and capacitor $C$ or constant phase element connected in parallel. The structure and quality of diamond films were analyzed by scanning electron microscopy and the Raman spectroscopy. The impedance measurements showed that concentration of non-diamond admixture has essential influence on electrochemical properties of diamond layers.
\end{abstract}

DOI: $10.12693 /$ APhysPolA.128.447

PACS: 81.05.Uw, 81.07.-b, 78.66.-w

\section{Introduction}

Diamond films synthesized by chemical vapor deposition (CVD) have great potential for a variety of applications including microelectronic, optics, electrochemistry and recently also in sensor applications [1]. Such broad range of applications is possible because the diamond films possess unique properties such as large energy band gap, high carrier mobility, high breakdown voltage, high thermal conductivity, resistance to high temperature and radiation [2].

Diamond films are recently considered to be an ideal electrode material for electroanalysis and electrolysis applications [3, 4]. When treated with hydrogenplasma, the surface of diamond film becomes hydrogenterminated and shows $p$-type surface conductivity [5].

The electrical properties of the CVD diamond films differ significantly from the single crystal diamond because of grain boundaries and other structural defects. In crystalline materials, there is a well-defined band gap with the possibility of additional states (donor or acceptor) lying within the gap.

The electrical properties of undoped polycrystalline diamond (PCD) have been studied by many researchers $[6,7]$ and different mechanisms of conduction have been proposed. According to Sugino et al. [8] the conduction in diamond films can be described by three independent mechanisms: frequency-independent

\footnotetext{
* corresponding author; e-mail:

miroslaw.szybowicz@put.poznan.pl
}

band conduction, hopping conduction, and frequencydependent conduction.

Conte et al. have shown that the currents flow predominantly on grain boundaries by hopping [9]. Above is in accordance with earlier studies, which have also show that the grain boundaries within the films were the main conduction path in undoped polycrystalline diamond films $[6,7]$.

One of the promising application of diamond in field effect transistors (ISFET), can be realized when an electrolyte takes the role of the gate contact between two ohmic source and drain contacts (solution gate field effect transistor (SGFET)) [10].

This application is usually based on the unique property of undoped diamond to acquire a pronounced surface conductivity that develops when the hydrogen terminated surface is exposed to humid air [11].

The conduction mechanisms involved in polycrystalline diamond films are still a subject of discussion, especially in the low frequency regime. In the present work, we have used impedance spectroscopy to study the conduction mechanisms in diamond films in the frequency range of $0.01-10^{5} \mathrm{~Hz}$. It is supposed that hopping across the grain boundaries can be the dominant mechanism above $10 \mathrm{~Hz}$ [12]. Below $10 \mathrm{~Hz}$, there is significant contribution to conduction from the interface states between the crystalline grains. An electrical model has been proposed for the various conduction mechanisms in the CVD diamond films and the results are explained based on this model.

\section{Experimental details}

The diamond films growth rate was $0.2-0.8 \mu \mathrm{m} / \mathrm{h}$ on 
ultrasonically cleaned, $0.5 \mathrm{~mm}$ in diameter tungsten wire as substrates by high frequency CVD (HF CVD) technique. The stainless-steel chamber was water-cooled. The total pressure during growth was 60 mbar. Deposition was carried out using methane as the carbon containing gas, diluted in $\mathrm{H}_{2}$. The total gas flow rate was fixed at the $100 \mathrm{sccm}$ and the percentage flows for $\mathrm{CH}_{4}$ and $\mathrm{H}_{2}$ were 1 and 3 vol.\%, respectively. Prior to growth, the substrates were seeded with $1 \mu \mathrm{m}$ diamond powder in an ultrasonic bath. The growth temperature was estimated to be $\approx 750{ }^{\circ} \mathrm{C}$ and the thickness of the films varied between $2-3 \mu \mathrm{m}$. Further details of the deposition system are available from an earlier publication [13].

The Raman spectra were recorded at room temperature in back scattering geometry using Renishaw inVia Raman spectrometer. A tunable Ar ion laser was used for $488 \mathrm{~nm}$ as an excitation source. The laser beam was tightly focused on the sample surface through a Leica 50× LWD microscope objective (LWD - long working distance) with numerical aperture (NA) equals to 0.5 leading to a laser beam diameter about $2 \mu \mathrm{m}$. Spectral resolution was about $\pm 2 \mathrm{~cm}^{-1}$. The Raman scattering spectra of diamond CVD layers were investigated in the spectral range of $1000-2000 \mathrm{~cm}^{-1}$. All data collection were analyzed using Renishaw WiRE 3.1 software using curve fitting method.

The impedance measurements were performed in $0.1 \mathrm{M}$ $\mathrm{KCl}$ electrolyte at the potential of $0 \mathrm{~V}$ versus $\mathrm{Ag} / \mathrm{AgCl}$. The EIS spectra were recorded in frequency range of $0.1-10^{6} \mathrm{~Hz}$ were recorded using HIOKI $3532 \mathrm{LCR}$ HiTester. The measurements were performed at room temperature. The obtained electrodes are fully closed and encapsulated as it was checked by using EDS and Raman mapping. For impedance modeling the "EIS Spectrum Analyser" software was used.

\section{Results and discussion}

The surface morphology of the investigated diamond films was found to depend on the concentration of methane vapor in the working gas. With increasing methane concentration diamond film morphology changes from well faceted structure (Fig. 1a), with
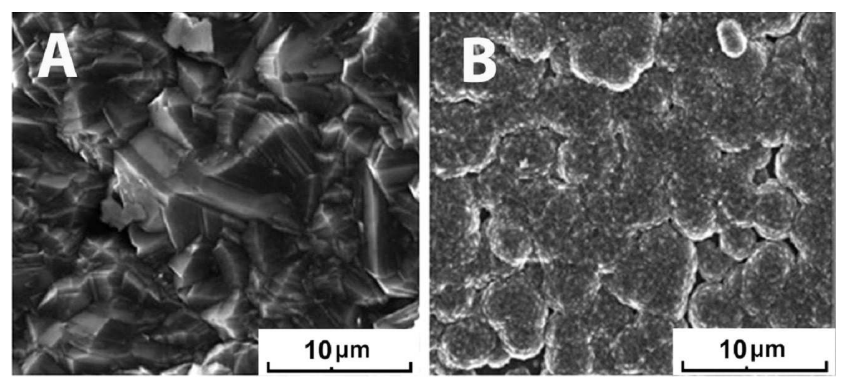

Fig. 1. SEM photos of diamond films synthesized at different concentrations of methane in the working gas: A $1 \%$ and B $3 \%$. crystallites sizes of the order of $8 \mu \mathrm{m}$ to the ball-like structure with no well pronounced crystalline features i.e. the diamond film morphology tends to be less faceted.

In order to estimate diamond film quality the Raman spectroscopy was used. This technique is widely used as a diagnostic tool for evaluation of diamond and chemical vapor deposited diamond films [14]. The diamond crystal is characterized by single sharp Raman line at $1332.5 \mathrm{~cm}^{-1}$ with full width at half maximum equal to $2-2.5 \mathrm{~cm}^{-1}[15]$. In the case diamond synthesis via CVD process except much broader Raman diamond line, the broad band at $1500-1600 \mathrm{~cm}^{-1}$ ( $G$-band) which indicates on co-existence of amorphous carbon phase in CVD diamond layer. The Raman spectra of the diamond films from Fig. 1 are presented in Fig. 2.

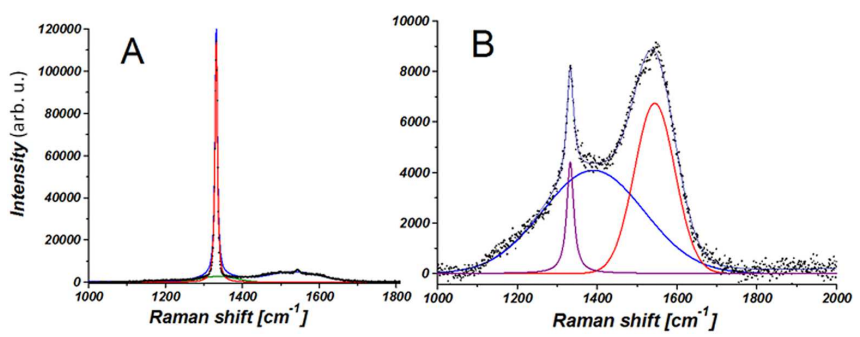

Fig. 2. Raman spectra of diamond films presented in Fig. 1.

As it seen from Fig. 2 in all Raman spectra $G$-band from amorphous carbon admixture is observed. The admixture of the amorphous carbon phase in CVD diamond film has crucial influence on diamond quality which can be described by so-called quality factor $f_{\mathrm{g}}$ defined as $[16,17]$ :

$$
f_{\mathrm{g}}=\frac{75 I_{d}}{75 I_{d}+\sum_{n d} I_{n d}},
$$

where $I_{d}$ is the diamond Raman line integrated intensity and $\Sigma_{n d} I_{n d}$ is the sum of the Raman peaks associated with amorphous carbon phase admixture ( $D$ and $G$-line). The factor 75 takes into account the more effective Raman scattering by amorphous carbon phase [18].

Another criterion which allows for comparing qualities of different diamond films is full width at half maximum (FWHM) of the first-order diamond Raman line and according to general convention the smaller FWHM, the better quality diamond film [19, 20]. In order to evaluate diamond quality each Raman spectrum was deconvoluted into pure diamond and amorphous carbon Raman bands according to procedure described earlier [13]. The obtained results are collected in Table I.

The data collected in Table I clearly indicate that the diamond layers have very different qualities as described by FWHM of diamond Raman lines and by quality factor $f_{\mathrm{g}}$ as well. 
TABLE I

Parameters of Raman spectra from Fig. 2.

\begin{tabular}{c|c|c|c|c|c|c|c}
\hline \hline Sample & $\begin{array}{c}\text { FWHM } \\
\text { Diamond }\left[\mathrm{cm}^{-1}\right]\end{array}$ & $\begin{array}{c}\text { Diamond peak } \\
\text { position }\left[\mathrm{cm}^{-1}\right]\end{array}$ & $\begin{array}{c}\text { FWHM } \\
D \text {-band }\left[\mathrm{cm}^{-1}\right]\end{array}$ & $\begin{array}{c}D \text {-peak position } \\
{\left[\mathrm{cm}^{-1}\right]}\end{array}$ & $\begin{array}{c}\text { FWHM } \\
G \text {-band }\left[\mathrm{cm}^{-1}\right]\end{array}$ & $\begin{array}{c}G \text {-peak position } \\
{\left[\mathrm{cm}^{-1}\right]}\end{array}$ & $\begin{array}{c}\text { Quality } \\
\text { factor } f_{\mathrm{g}}\end{array}$ \\
\hline A & 7 & 1332 & 110 & 1337 & 160 & 1531 & 0.999 \\
B & 21 & 1333 & 299 & 1390 & 120 & 1554 & 0.975
\end{tabular}

TABLE II

The parameters from simulation.

\begin{tabular}{c|c|c|c|c|c|c|c|c|c}
\hline \hline Sample & $R_{1}[\Omega]$ & $R_{2}[\Omega]$ & $\begin{array}{c}\mathrm{CPE}_{1} \\
{\left[\mathrm{~F}^{\alpha} \Omega^{\alpha-1} \mathrm{~cm}^{-2}\right]}\end{array}$ & $\alpha_{1}$ & $R_{3}[\Omega]$ & $\begin{array}{c}\mathrm{CPE}_{2} \\
{\left[\mathrm{~F}^{\alpha} \Omega^{\alpha-1} \mathrm{~cm}^{-2}\right]}\end{array}$ & $\alpha_{2}$ & $R_{4}[\Omega]$ & $\begin{array}{c}C_{1} \\
{\left[\mathrm{~F} \mathrm{~cm}^{-2}\right]}\end{array}$ \\
\hline $\mathrm{A}$ & 310 & $2.03 \times 10^{4}$ & $3.8 \times 10^{-10}$ & 0.79 & $2.1 \times 10^{4}$ & $1.72 \times 10^{-9}$ & 0.93 & $5.6 \times 10^{5}$ & $2.8 \times 10^{-8}$ \\
$\mathrm{~B}$ & 140 & $514 \times 10^{4}$ & $4.5 \times 10^{-6}$ & 0.99 & $122 \times 10^{5}$ & $41 \times 10^{-6}$ & 0.99 & $3.2 \times 10^{4}$ & $96 \times 10^{-6}$
\end{tabular}

The electrochemical response of diamond electrode was studied by electrochemical impedance spectroscopy (EIS). Figure 3 shows the Nyquist plot at polycrystalline diamond electrodes. The fitting quality is well defined by solid line in Fig. 3 (theoretical calculations) and squares are experimental points.

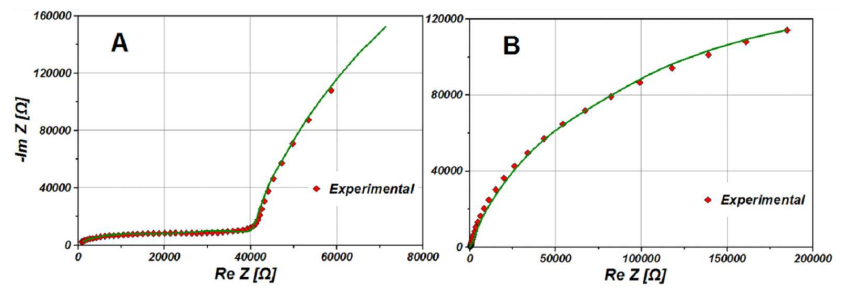

Fig. 3. The Nyquist plot at polycrystalline diamond electrodes.

The presented spectra at high frequency range are very different but at low frequencies are characterized by a tilted lines. The low frequency line are not vertical indicating a non-ideal polarization. The tilted angle is attributed to the inhomogeneous porous structure [21].

In the case of A electrode their Nyquist plot displays a flattened high frequency semicircle associated probably with the contact impedance resulted from electrical connection between diamond microcrystals as well as that between microcrystals and the backing tungsten wire [22].

Due to much smaller crystallite sizes (the sizes in $\mathrm{nm}$ range) in the case of $\mathrm{B}$ electrode the contact between diamond layer and the backing tungsten wire can be much better.

EIS spectra generally provide the data on electrode capacitance and charge-transfer kinetics. An electrode interface resembles an electronic circuit describing its impedance. In the case of standard electrodes $(\mathrm{Au}, \mathrm{Pt}$, etc.) one of the simplest circuit which can represent electrode/solution interface is, in general, a Randles circuit [23].
The CVD diamond layer deposited on conductive substrate from electrochemical point of view is more complicated system comparing to the standard situation (standard electrodes) and proposed equivalent circuit should describe the electrolyte/diamond interface, the capacitance and resistance of diamond layer and finally diamond/substrate interface.

Therefore, the proposed equivalent circuit shown in Fig. 4 used to simulate the impedance spectrum of the diamond electrodes consists of three impedance components $Z_{1}\left(R_{2} / \mathrm{CPE}_{1}\right), Z_{2}\left(R_{3} / \mathrm{CPE}_{2}\right), Z_{3}\left(R_{4} / C_{1}\right)$ and additionally ohmic resistance $R_{1}$ of the bulk solution is also added. Equivalent circuit for the electrical response of a polycrystalline sample showing contributions from the grain interiors: $Z_{1}\left(R_{2} / \mathrm{CPE}_{1}\right)$, grain boundaries $Z_{2}\left(R_{3} / \mathrm{CPE}_{2}\right)$, and electrolyte/electrode interface $Z_{3}\left(R_{4} / C_{1}\right)$.

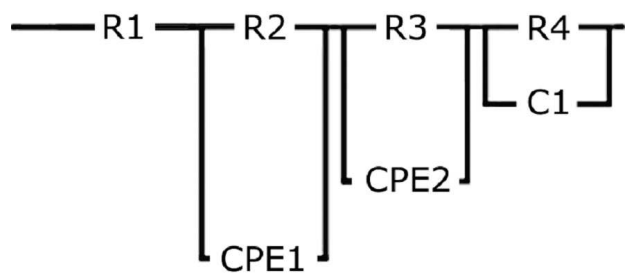

Fig. 4. The proposed equivalent circuit to describe the reaction at polycrystalline diamond electrode.

The results of simulation by using above model are presented in Fig. 3 and are shown by solid lines and obtained parameters are collected in Table II.

Considering the rough or porous electrode surface, both the contact capacitance $\mathrm{CPE}_{1}$ and low-frequency capacitance $\mathrm{CPE}_{2}$ are described by a constant phase element (CPE). The CPE is defined as:

$$
Z_{\mathrm{CPE}}=(\sigma)^{-1}(\mathrm{j} \omega)^{-\alpha},
$$

where $\sigma$ and $\alpha$ are frequency-independent constants and $\omega$ is the angular frequency. The exponent $\alpha$ is a factor ranging between 0 and 1 (value 1 corresponds to the ca- 
pacitor) and its physical meaning can be related to the surface roughness of the electrode surface.

When analyzing the data from Table II one can notice that main differences in the electrodes depends mainly on their capacitance values which are $2-4$ orders bigger in the case of $\mathrm{B}$ electrode comparing to the appropriate values of $A$ electrode.

Very often the frequency dependent admittance is used to study the involvement in transport mechanisms of electronic states associated to defects at grain boundaries (GB), or at the grain surface, in respect of those internal to the grain itself, as well as to carriers displacement inside the connective tissue in the form of amorphous carbon phase. For interpretation of experimental observations we will use the real part of admittance. In the case of homogeneous system, the admittance, $Y$, is given by the following relation [24]:

$$
Y(\vartheta, T)=G(\vartheta, T)+\mathrm{j} B(\vartheta T),
$$

where $G(\nu, T)$ and $B(\nu, T)$ are the temperatureand frequency-dependent conductance $(G)$ and susceptance $(B)$, respectively.

The frequency-dependent conductance spectra of our diamond layers are reported in Fig. 5.
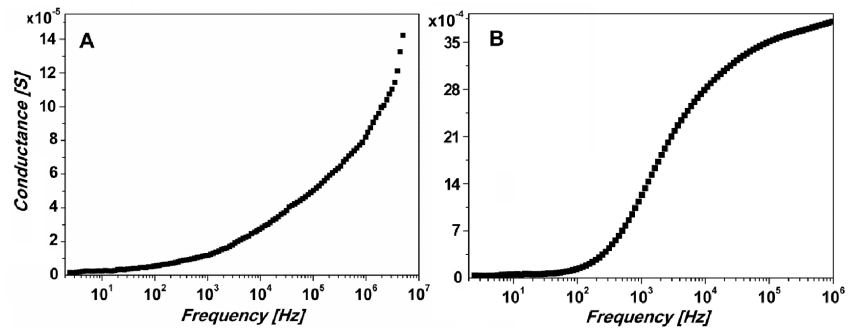

Fig. 5. Frequency-dependent conductance of the diamond layers A and B, respectively.

Both curves show a similar behavior: in the low frequency regime, conductance is independent of frequency to certain frequency $\vartheta_{c}$ whereas above this frequency the signal increases following the formula [25]:

$$
G(\vartheta)=G_{0}\left[1+\left(\frac{\vartheta}{\vartheta_{\mathrm{c}}}\right)^{n}\right]+A \vartheta^{m},
$$

where $A$ is adaptive term, $n$ is ranging between 0 and 1 and $m \geq 1$. $G$ is associated to the DC conductivity in the percolating connective tissue also involving GBs. $G$ describes the low-frequency conductivity relaxation where charge carriers displacement takes place mainly by hopping among defective sites at the Fermi level. Increasing frequency, the conductivity shows a dispersive regime which is related to charge carriers relaxation inside potential wells, when their energies are not sufficient to activate a conduction mechanism with a real charge displacement. The second term in Eq. (4), is an approximation, which describes the transition between the two above mentioned relaxation behaviors.

As it is seen from Fig. 5 the frequency $\vartheta_{\mathrm{c}}$ at which the conductance starts to be dispersive strongly depends on diamond layers crystalline size.
In the case of larger microcrystals (sample A) $\vartheta_{\mathrm{c}}$ is equal about $10^{4} \mathrm{~Hz}$ and when diamond layer has nanodiamond structure $\vartheta_{\mathrm{c}}$ has much lower value and is equal to about $100 \mathrm{~Hz}$.

The frequency-independent regime for conductance spans on a wider range of frequencies below $\nu_{\mathrm{c}}$ i.e. before the dispersive regime set-on for sample $\mathrm{A}$ in comparison to sample B. It means charge carriers displacement takes place mainly by hopping among defective sites at the Fermi level.

\section{Conclusions}

The polycrystalline diamond films deposited on polished tungsten substrates using methanol and hydrogen by HFCVD have been synthesized. SEM and Raman measurements showed that we have obtained diamond films with varying quality and with different concentration of amorphous carbon admixture.

The EIS spectra of HFCVD diamond layers was studied in the frequency range of $0.1-10^{6} \mathrm{~Hz}$ at room temperature. An electrical equivalent circuit, which gives a very good fit for the impedance data, has been proposed. The proposed model indicates on two mechanisms contributing to electrical conduction. The major mechanism is the hopping of carriers at the grain boundaries. In addition to hopping, there is a contribution of interface states at the low-frequency region.

The amorphous carbon admixture in diamond layers is an important factor determining their electrochemical properties.

\section{Acknowledgments}

This work was supported in part by the Research Project of Poznań University of Technology 06/62/DSPB/0415/2015 and in cooperation with MWiK in Bydgoszcz and B\&B Stal in Grębocin/Toruń.

\section{References}

[1] M.W. Varney, D.M. Aslam, A. Janoudi, H.Y. Chan, D.H. Wang, Biosensors 1, 118 (2011).

[2] P. Calvani, A. Corsaro, F. Sinisi, M.C. Rossi, G. Conte, E. Giovine, W.'Ciccognani, E. Limiti, $M i$ crowave Opt. Technol. Lett. 51, 2786 (2009).

[3] J. Iniesta, P.A. Michaud, M. Panizza, C. Comninellis, Electrochem. Commun. 3, 346 (2001).

[4] R.I. Stefan, S.G. Bairu, Anal. Chem. 75, 5394 (2003).

[5] J. Ristein, W. Zhang, L. Ley, Phys. Rev. E 78, 041602 (2008).

[6] B.J. Lee, B.T. Ahn, J.K. Lee, Y.J. Baik, Diamond Relat. Mater. 10, 2174 (2001).

[7] P. Gonon, A. Deneuville, F. Fontaine, E. Gheeraert, J. Appl. Phys. 78, 7059 (1995).

[8] T. Sugino, Y. Muto, K. Karasutani, J. Shirafuji, K. Kobashi, Diamond Relat. Mater. 2, 803 (1993). 
[9] G. Conte, M.C. Rossi, F. Spaziani, R. Arcangeli, Diamond Relat. Mater. 14, 570 (2005).

[10] H. Kawarada, Y. Araki, T. Sakai, T. Ogawa, H. Umezawa, Phys. Status Solidi A 185, 79 (2001).

[11] F. Maier, M. Riedel, B. Mantel, J. Ristein, L. Ley, Phys. Rev. Lett. 85, 3472 (2000).

[12] K.G. Girija, C.A. Betty, Diamond Relat. Mater. 13, $1812(2004)$.

[13] K. Fabisiak, A. Banaszak, M. Kaczmarski, M. Kozanecki, Opt. Mater. 28, 106 (2006).
[14] K. Fabisiak, R. Torz-Piotrowska, E. Staryga, M. Szybowicz, K. Paprocki, A. Banaszak, P. Popielarski, Mater. Sci. Eng. B 177, 1352 (2012).

[15] S. Prawer, R.J. Nemanich, Philos. Trans. R. Soc. A 362, 2537 (2004).

[16] S.R. Sails, D.J. Gardiner, M. Bowden, J. Savage, D. Rodway, Diamond Relat. Mater. 5, 589 (1996).

[17] K.M. McNamara, K.K. Gleason, D.J. Vestyck, J.E. Butler, Diamond Relat. Mater. 1, 1145 (1992). 